\title{
A New Technique for Foot-Off and Foot Contact Detection in a Gait Cycle Based on the Knee Joint Angle Using Microsoft Kinect v2
}

\author{
A. Amini, Member, IEEE EMBS, K. Banitsas, Member, IEEE, S. Hosseinzadeh, Member, IEEE
}

\begin{abstract}
The Microsoft Kinect RGB-D sensor has been proven to be a reliable tool for gait analysis and rehabilitation purposes. Although it is accurate for detecting upper body part movements, even the second iteration of the Kinect sensor lacks the accuracy when it comes to lower extremities. while detecting foot-off and foot contact phases of a gait cycle is an important part of a gait performance analysis, The Kinect's intrinsic inaccuracies make it an unreliable tool to detect them accurately. We propose a new Kinect based technique for detecting foot-off and foot contact phases in a gait cycle that solely relies on a subject's knee joint relative angle. The system was tested on 11 healthy subjects walking in pre-defined pathways in 12 walking sessions while the Kinect v2 camera was placed at different heights ranging from 0.65 to 1.57 and angles ranging from 0 to 45 degrees to the ground. The algorithm's accuracy was also compared to another footstep detection method based on the subject's ankle joints height to the ground. The results showed $86.52 \%$ accuracy in detecting foot-off and foot contact events on average for both feet.
\end{abstract}

\section{INTRODUCTION}

Footstep detection is an important measurement in rehabilitation and gait analysis studies, as many disorders feature symptoms that directly or indirectly affect patients' gait cycle and walking style. There are different techniques used in detecting footsteps and evaluating gait cycles based on on-body sensors that, although accurate, they can affect the subject's walking style and consequently, the data reading as the subject must wear special clothing embedded with on-body sensors during the gait performance analysis. As an alternative approach to the aforementioned technique, one can consider the employment of unobtrusive depth cameras such as Microsoft Kinect v2. As the Kinect was designed as a replacement for conventional game controllers, it is very effective in reading body joints data, especially from upper body parts that are more active in a gaming session. Nevertheless, due to the Kinect's intrinsic inaccuracies in data acquisition, particularly for lower extremities [1], innovative approaches have been made to compensate these issues (further discussed in the Background section of this paper). Moreover, due to the nature of some degenerative diseases such as Parkinson's disease that feature gait related symptoms including Freezing of Gait (FOG), minor inaccuracies either greatly

Mr Amin Amini, Dr. Konstantinos Banitsas are with the College of Engineering Design and Physical Sciences, Department of Electronics and Computer Engineering, Brunel University London, UK (amin.amini, konstantinos.banitsas @brunel.ac.uk). Mr. Salaheddin Hosseinzadeh is with the School of Engineering \& Built Environment, Department of Computer, Communications and Interactive Systems, Glasgow Caledonian University, UK. salaheddin.hosseinzadeh@gmail.com. affect the data collection or render the entire acquired data unusable.

This study proposes a new approach that is solely based on the subject's knee joint angle to determine foot-offs and foot contacts, regardless of the changes of signal acquisition accuracy due to the subject's location or distance to the camera in a 3-D environment.

\section{BACKGROUND}

\section{A. The Kinect $v 2$}

The Microsoft Kinect v2 is the second iteration of the Kinect series designed for the Xbox One gaming console as a replacement for conventional gamepads. It is a Time-offlight (ToF) camera featuring a 1080p RGB sensor alongside a $424 p$ depth/infra-red sensor running at an average of 30 frames per second [2]. The Kinect v2 has many improvements compared to its predecessor such as the ability to track 25 joints of up to six subjects simultaneously. The camera has a wider field of view that makes it easier to use in smaller and narrower areas. Due to these enhancements, the accuracy of data collection, especially in capturing skeletal information, has been significantly increased. Consequently, the Kinect v2 has proven to be an ideal device for gait performance analysis and evaluation due to its higher accuracy and low implementation cost.

\section{B. State of the Art}

Computer-vision based techniques are the preferred method for gait performance analysis [3-8], as there is no need for on-body sensor attachments that might in turn, affect the data collection during a gait cycle and make it harder for the subjects to use. Nonetheless, there are disadvantages to this method such as higher computational power required for signal analysis and image processing and intrinsic data acquisition inaccuracies, especially in Kinect sensors [2].

Consequently, in Kinect-based gait cycle detection studies, researchers had to implement rather innovative tactics in order to compensate for the aforementioned issues. For instance, in [9], the distance between subjects' knees joints center along the longitudinal walking axis were calculated in order to estimate the heel strikes. In order to eliminate the increase of Kinect joint localization inaccuracies in further distances to the camera [6], subjects were asked to walk on a treadmill while knee joint height was estimated according to anthropometric data.

Also in [10], subjects were asked to walk on a treadmill in order to keep their distance to the Kinect the same at all times during a gait cycle. In [11], in order to minimize data acquisition inaccuracies, a series of Kinect sensors were 
located at different locations alongside the subject's walking path. This approach, although has managed to rectify the Kinect's inaccuracy issue, includes the implementation of four Kinect sensors that have to be placed carefully at certain distances to each other that might introduce more limitation in real-life practices. In another attempt [12], the subject was asked to walk inside a cart while a Kinect was mounted at the bottom, facing the subject to keep the subject's distance to the camera constant, which might affect the walking style and gait performance of the subject.

This research on the other hand, follows a different approach to detect foot-offs and foot contacts that relies only on the changes of the subject's knee joints angle during a gait cycle.

\section{SYSTEM ARCHITECTURE}

The proposed algorithm was implemented in Microsoft Visual Studio 2015 Community Edition environment using Visual C\# language.

\section{A. Angle Determination}

As studies previously noted, the Kinect skeletal joints relative $3-\mathrm{D}$ coordinates data reading are less susceptible to noise and inaccuracies compared to their distance to the ground data acquisition [4,13-15]. Thus, for each leg, a knee joint angle was determined by considering the location of the neighboring joints such as hip and ankle in the Cartesian coordinate. The hip, the knee and the ankle position in Cartesian space are defined with three vectors, with the
Kinect being at the origin of the 3-D space. This vector definition is formulated in Equation 1.

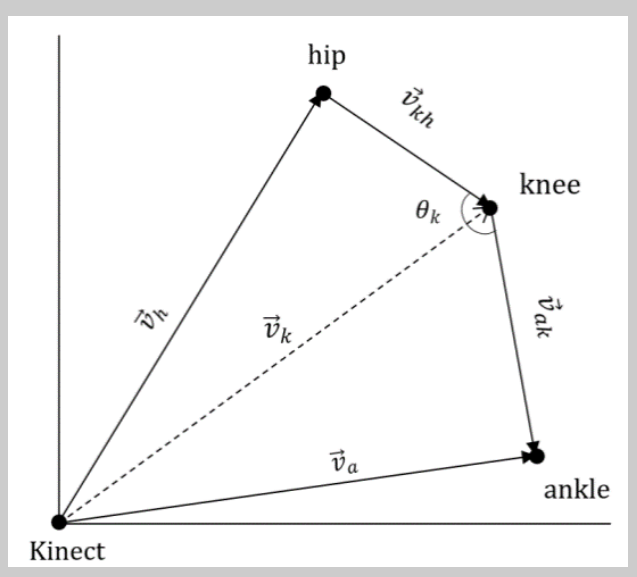

Figure 1. Determination of $\theta$ using hip and ankle joints

$$
\begin{gathered}
\vec{v}_{k h}=\vec{v}_{k}-\vec{v}_{h} \\
\vec{v}_{a k}=\vec{v}_{a}-\vec{v}_{k} \\
\theta_{k}=\cos ^{-1}\left(\vec{u}_{k h} \cdot \vec{u}_{a k}\right)
\end{gathered}
$$

Equation 1. Knee joint 3-D angle determination

Were $\overrightarrow{\boldsymbol{v}}_{\boldsymbol{k} \boldsymbol{h}}$ and $\overrightarrow{\boldsymbol{v}}_{\boldsymbol{a} \boldsymbol{k}}$ are the 3 -D vectors connecting the subject's hip to the knee and knee to the ankle, respectively that is also depicted in Figure 1. Moreover, $\overrightarrow{\boldsymbol{u}}_{\boldsymbol{k} \boldsymbol{h}}$ and $\overrightarrow{\boldsymbol{u}}_{\boldsymbol{a} \boldsymbol{k}}$ are the unit vectors of $\overrightarrow{\boldsymbol{v}}_{\boldsymbol{k} \boldsymbol{h}}$ and $\overrightarrow{\boldsymbol{v}}_{\boldsymbol{a} \boldsymbol{k}}$, respectively.

\section{B. Step Detection Algorithm}

A foot-off event is considered to have occurred when the knee angle of one foot has decreased to less than a particular threshold, which was experimentally acquired throughout the initial experiments (170 degrees). Moreover, a foot contact is triggered when the knee angle of the same foot has returned to its original value in a time period of more than 200 milliseconds. The $200 \mathrm{~ms}$ timing threshold was set to avoid the false positives flag ups due to the Kinect inconsistencies and noise.

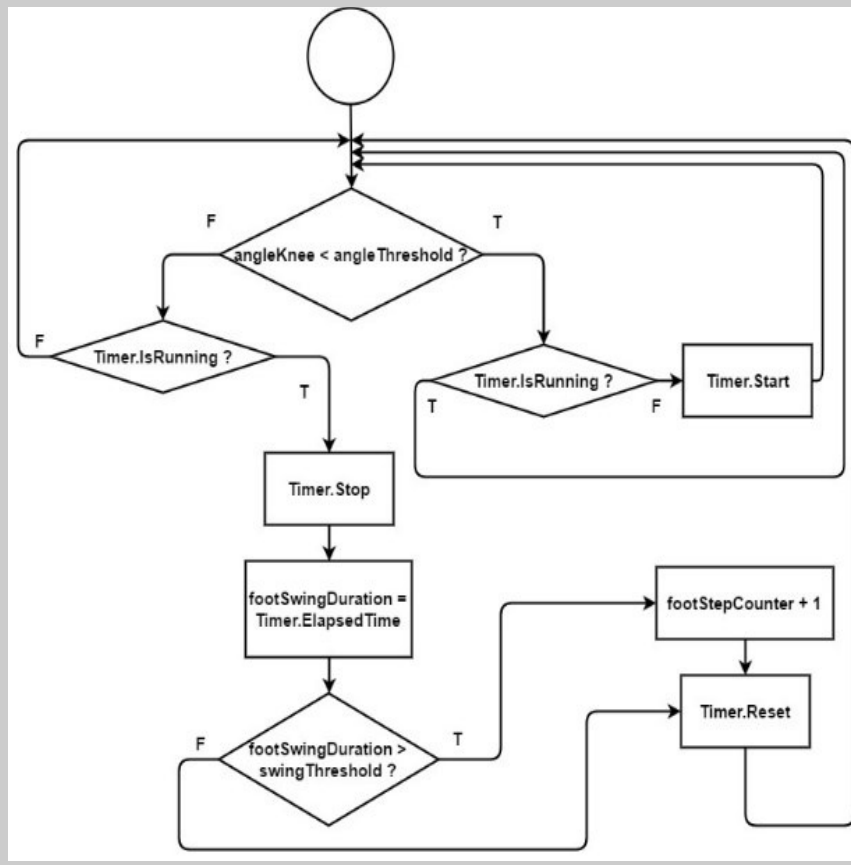

Figure 2. Step detection (foot-off and foot contact) process flowchart

Figure 2 demonstrates the subject's footstep detection process including foot-offs and foot contacts. The algorithm loops at approximately 30 frames per second while the Kinect camera is tracking the subject.

Moreover, in order to evaluate the algorithm's accuracy, another footstep detection algorithm was developed based on the subject's ankle distance-to-ground. In this method, a foot-off is considered to have occurred when a foot's ankle joint 3-D Euclidean distance from the ground has increased, compared to the pre-measured ankle's height. Consequently, a foot contact occurs when the value for the same foot ankle distance-to-the-ground has return back to the same value in a time period of more than 250 milliseconds.

$$
d=\frac{A x+B y+C z+D}{\sqrt{A^{2}+B^{2}+C^{2}}}
$$

Equation 2. Knee joint 3-D angle determination

Where $\boldsymbol{x}, \boldsymbol{y}$ and $\boldsymbol{z}$ are the 3-D coordinates of a joint.

The subjects' ankles were chosen for this method, because among human lower extremities, the ankle joint is less susceptible to noise and inaccuracies compared to other joints such as foot [1]. 


\section{EXPERIMENT METHODS}

\section{A. Testing Environment}

A testing environment was used to carry out the trials as below:

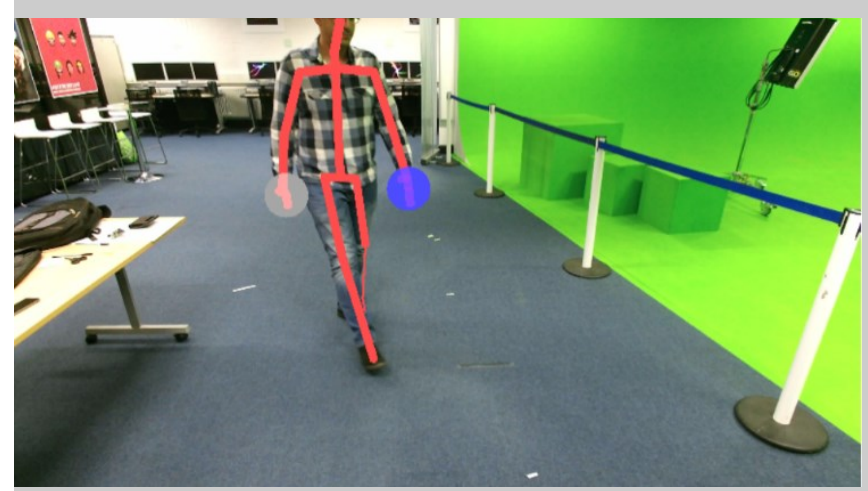

Figure 3. The testing environment: the subject is walking diagonally towards the Kinect camera while his body joints are being tracked

The Kinect sensor was setup at different angles ranging from 0 to $45(0,10,22$ and 45$)$ degrees to the ground and at different heights ranging from 0.65 to $1.57(0.65,1$ and 1.57 meters) in order to identify any possible differentiation in results based on the camera angle and height factor.

\section{B. Test Cases}

For the testing trial, 11 subjects participated by walking in pre-determined paths in 12 walking sessions including diagonally walking towards the camera, while their body
Kinect v2. The subjects' characterist were as follows:

TABLE 1. Test subjects' characteristics ( $n=7 ; 4$ males, 3 females)

\begin{tabular}{|c|c|c|}
\hline Subject Characteristics & Range & $\begin{array}{l}\text { Standard } \\
\text { Deviation }\end{array}$ \\
\hline Age & $24-31$ & 2.34 \\
\hline Height (cm) & $163-187$ & 8.31 \\
\hline Weight (kg) & $51-100$ & 16.35 \\
\hline BMI (kg/m $\mathbf{2})$ & $17.3-30.1$ & 3.83 \\
\hline
\end{tabular}

\section{Results}

Eleven subjects were asked to walk in pre-determined paths while their skeletal data was captured by Kinect v2, which was placed at different heights and angles to the ground. Figure 4 illustrates a subject's walking session and knee joints behavior during a gait cycle. It shows that in a standstill pose or during foot contact phase, the knee joint angle remains approximately at 176 degrees. The acquired signal required no further processing as it had low SNR for gait performance analysis resulting in a low latency, lowresource consumption footstep detection.

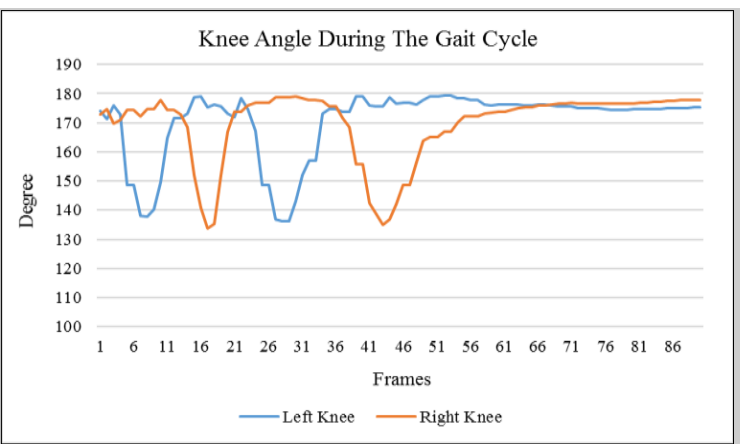

Figure 4. Knee joint angle value during a gait cycle

Figure 5 shows the same subject's walking session, walking towards the Kinect v2 camera. It indicates that the knee joint angle reading remained unaffected by the joint's distance-to-Kinect changes, as it is relative to the subject's skeletal joints. The subject's right knee data was omitted in the figure for simplicity.

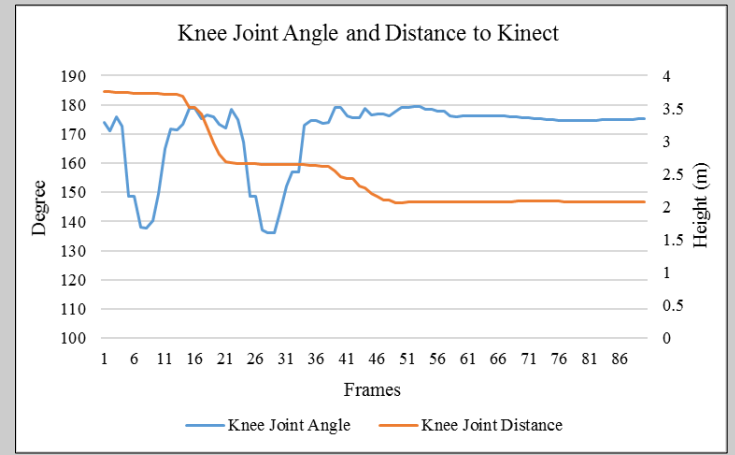

Figure 5. Knee joint angle and its distance to the camera during a gait cycle

The Knee joints angle performance during a gait cycle was compared against a different footstep method based on the subject's ankle joints distance-to-ground, in order to evaluate how the footstep detection accuracy has improved. The following figure (figure 6) shows the same walking session based on the subject's ankle joint distance-toground.

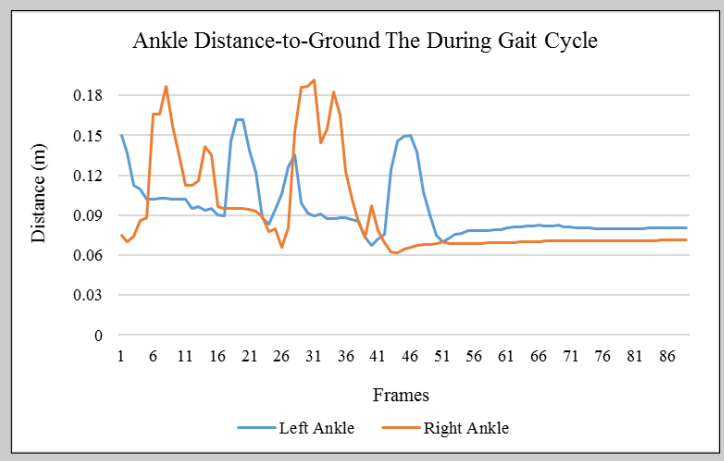

Figure 6. Ankle joint distance-to-ground value during a gait cycle

As Figure 6 illustrates, not only joints height to the ground detection by the Kinect $\mathrm{v} 2$ is noisy and less accurate, but also inconsistent and highly dependent on subject's 
distance to the camera due to the Kinect's aforementioned issues.

As mentioned previously, the Kinect v2 camera was placed at different distances and angles compared to the ground plane. It was observed that different heights from the ground (including 0.65, 1 and 1.57 meters) did not have any effect on the knee joint angle measurement as long as the subject was within the Kinect v2 detection range. Different Kinect camera angles $(0,10,22 \& 45$ degrees compared to the ground plane) were also studied, in order to determine the possibility of different outcomes. It was concluded that similar to the Kinect's height, the camera's angle did not have a significant effect on the measurement of the knee joint angle. Nonetheless, it was observed that as soon as a knee's next closest joint (such as hip or ankle) becomes undetected due to an obstruction or limited field of view, the knee joint angle reading becomes unreliable. Thus, this study did not cover the effect of angles larger than 45 degrees to the ground due to the Kinect's limited field of view.

It was also concluded that the footstep detection using solely the knee joint angle is a reliable method to detect foot-off and foot contact phases of a gait cycle. The system showed $86.37 \%$ and $86.67 \%$ accuracy for left and right foot, respectively, compared to the ankle joint-to-ground detection algorithm accuracy of $43.65 \%$ and $42.06 \%$ for left and right foot, respectively. Moreover, the proposed method had less footstep detection latency $(200 \mathrm{~ms})$ compared to the $250 \mathrm{~ms}$ delay in the ankle joint-to-ground detection algorithm.

(n)

\section{CONCLUSION}

In this paper, we introduced a novel low-latency and low-resource approach in detecting footsteps including footoff and foot contact phases of a gait cycle based on a subject's knee joint angle. Eleven healthy subjects participated in the testing, including 12 different walking sessions. We investigated different conditions that might affect the angle measurement of knee joints such as the height of the Kinect camera, the angle of the Kinect camera to the ground, and the consistency and accuracy of the data acquisition in different distances to the camera. The algorithm's accuracy was also compared to another footstep detection method based on the subject's ankle joints height to the ground. It was concluded that neither the camera's height nor its angle to the ground has a significant impact on the data acquisition of the subject's knee joint angle, and as a result, on the step detection process. Nonetheless, the detection of the proposed system was limited to the Kinect v2 practical skeletal distance coverage (1.6 to 4 meters). Moreover, the system showed a consistent measurement, as long as none of the knee's neighboring joints (joints that are needed to be calculated for knee joint angle determination) is obstructed or undetected by the Kinect v2 camera.

Overall, due to the low latency and high accuracy of this technique and the fact that the system's accuracy is unaffected by the Kinect v2 intrinsic inaccuracies or its height or angle, it can be used for gait assessment scenarios that require a high level of accuracy, and the ability to detect subtle movements. Future research will be investigating the system's competency in detecting FOG incidents in Parkinson's disease patient that mandates fast and accurate footstep detection for gait performance analysis.

\section{REFERENCES}

[1] Q. Wang, Q. Wang, G. Kurillo, F. Ofli, and R. Bajcsy, "Evaluation of Pose Tracking Accuracy in the First and Second Generations of Microsoft Kinect Evaluation of Pose Tracking Accuracy in the First and Second Generations of Microsoft Kinect," in International Conference on Healthcare Informatics, 2015, pp. 380-389.

[2] A. Amini, K. Banitsas, and J. Cosmas, "A comparison between heuristic and machine learning techniques in fall detection using Kinect v2," in 2016 IEEE International Symposium on Medical Measurements and Applications (MeMeA), 2016, pp. 1-6.

[3] A. Amini Maghsoud Bigy, K. Banitsas, A. Badii, and J. Cosmas, "Recognition of Postures and Freezing of Gait in Parkinson's Disease Patients Using Microsoft Kinect Sensor," in 2015 7th International IEEE/EMBS Conference on Neural Engineering (NER), 2015, pp. 731-734.

[4] R. A. Clark, K. J. Bower, B. F. Mentiplay, K. Paterson, and Y. H. Pua, "Concurrent validity of the Microsoft Kinect for assessment of spatiotemporal gait variables," J. Biomech., vol. 46, no. 15, pp. 2722-2725, 2013.

[5] M. Gabel, R. Gilad-Bachrach, E. Renshaw, and A. Schuster, "Full Body Gait Analysis with Kinect *," in 2012 Annual International Conference of the IEEE Engineering in Medicine and Biology Society, 2012, pp. 1964-1967.

[6] B. Galna, G. Barry, D. Jackson, D. Mhiripiri, P. Olivier, and L. Rochester, "Accuracy of the Microsoft Kinect sensor for measuring movement in people with Parkinson's disease.," Gait Posture, vol. 39, no. 4, pp. 1062-1068, 2014.

[7] A. Procházka, O. Vyšata, M. Vališ, O. `Tupa, M. Schätz, and V. Ma rík, "Use of the image and depth sensors of the Microsoft Kinect for the detection of gait disorders," Neural Comput. Appl., vol. 26, no. 7, pp. 1621-1629, 2015.

[8] E. E. Stone and M. Skubic, "Unobtrusive, continuous, in-home gait measurement using the microsoft kinect," IEEE Trans. Biomed. Eng., vol. 60, no. 10, pp. 2925-2932, 2013.

[9] E. Auvinet, F. Multon, C. E. Aubin, J. Meunier, and M. Raison, "Detection of gait cycles in treadmill walking using a Kinect," Gait Posture, vol. 41, no. 2, pp. 722-725, 2015.

[10] X. Xu, R. W. McGorry, L. S. Chou, J. hua Lin, and C. chi Chang, "Accuracy of the Microsoft Kinect ${ }^{\mathrm{TM}}$ for measuring gait parameters during treadmill walking," Gait Posture, vol. 42, no. 2, pp. 145-151, 2015.

[11] D. J. Geerse, B. H. Coolen, and M. Roerdink, "Kinematic validation of a multi-Kinect v2 instrumented 10-meter walkway for quantitative gait assessments," PLoS One, vol. 10, no. 10, pp. 1-15, 2015.

[12] B. Sun, X. Liu, X. Wu, and H. Wang, "Human gait modeling and gait analysis based on Kinect," Proc. - IEEE Int. Conf. Robot. Autom., pp. 3173-3178, 2014.

[13] A. Corti, S. Giancola, G. Mainetti, and R. Sala, "A metrological characterization of the Kinect V2 time-of-flight camera," Rob. Auton. Syst., vol. 75, pp. 584-594, 2016.

[14] E. Stone and M. Skubic, "Evaluation of an inexpensive depth camera for in-home gait assessment," J. Ambient Intell. Smart Environ., vol. 3, no. 4, pp. 349-361, 2011.

[15] L. Yang, L. Zhang, H. Dong, A. Alelaiwi, and A. Saddik, "Evaluating and improving the depth accuracy of Kinect for Windows v2," IEEE Sens. J., vol. 15, no. 8, pp. 4275-4285, 2015. 